\title{
Increased frequency of temporal acoustic window failure in rheumatoid arthritis: a manifestation of altered bone metabolism?
}

Zsófia Kardos ${ }^{1 *}$, Csaba Oláh ${ }^{2,6^{*}}$, Mariann Sepsi ${ }^{3}$, Attila Sas ${ }^{4}$, László Kostyál ${ }^{3}$, Tünde Bóta ${ }^{3}$, Harjit Pal Bhattoa $^{5}$, Katalin Hodosi ${ }^{7}$, György Kerekes ${ }^{8}$, László Tamási ${ }^{1}$, Dániel Bereczki ${ }^{6,9}$, Zoltán Szekanecz ${ }^{7}$

Departments of ${ }^{1}$ Rheumatology, ${ }^{2}$ Neurosurgery, ${ }^{3}$ Radiology and ${ }^{4}$ Neurology, Borsod County Teaching Hospital, Miskolc, Hungary;

Departments of ${ }^{5}$ Laboratory Medicine, ${ }^{6}$ Neurology; Department of Medicine, Divisions of ${ }^{7}$ Rheumatology and ${ }^{8}$ Angiology, University of Debrecen Faculty of Medicine, Debrecen, Hungary;

${ }^{9}$ Department of Neurology, Semmelweis University, Budapest, Hungary

*Shared first authorship, equal contribution

None of the authors have any financial or other conflicts of interest.

Running title: Temporal acoustic windows in RA

Keywords: rheumatoid arthritis, biological therapy, temporal acoustic window failure, osteoporosis, BMD, osteoprotegerin

Correponding author

Zoltan Szekanecz MD, PhD

University of Debrecen Faculty of Medicine, Department of Medicine, Division of Rheumatology, Nagyerdei str 22, Debrecen, H-4032, Hungary

Phone: +36 52 255091, fax: +36 52 414969, email: szekanecz.zoltan@ med.unideb.hu 


\section{Abstract}

Objective. Assessment of intracranial vessels includes transcranial doppler (TCD). TCD performance requires intact temporal acoustic windows (TAW). Failure of TAW (TAWF) is present in $8-20 \%$ of people. There have been no reports on TAWF in rheumatoid arthritis (RA). Methods. Altogether 62 female RA patients were included. Among them, 20 were MTXtreated and biologic-free, 20 received infliximab and 22 tocilizumab. The controls included 60 non-RA women. TAWF, temporal bone thickness and texture were determined by ultrasound and CT. BMD and T-scores of multiple bones were determined by DEXA. Several bone biomarkers were assessed by ELISA.

Results. In RA, $54.8 \%$ of the patients had TAWF on at least one side. Neither TAW could be identified in $34 \%$ of RA subjects. In contrast, only $20.0 \%$ of control subjects had TAWF on either or both sides ( $\mathrm{p}<0.001$ ). In RA vs controls, $53.0 \%$ vs $2.9 \%$ of subjects exerted the trilayer, "sandwich-like" structure of TAW ( $<<0.001)$. Finally, in RA vs controls, the mean temporal bone thickness values of the right TAW were $3.58 \pm 1.43 \mathrm{~mm}$ vs $2.92 \pm 1.22 \mathrm{~mm}$ ( $\mathrm{p}=\mathrm{NS}$ ), while those of the left TAW were $4.16 \pm 1.56 \mathrm{~mm}$ vs $2.90 \pm 1.16 \mathrm{~mm}$ ( $\mathrm{p}=0.001)$. There was close association between TAWF, bone thickness and texture $(\mathrm{p}<0.05)$. These TAW parameters all correlated with age, however TAW failure and texture also correlated with serum osteoprotegerin. TAW bone thickness inversely correlated with hip BMD $(\mathrm{p}<0.05)$.

Conclusions. TAWF, thicker and heterogeneous temporal bones were associated with RA. These features have been associated with bone loss and OPG production. Bone loss seen in RA may result in OPG release and stimulation of bone formation around TAW. 


\section{Introduction}

Rheumatoid arthritis (RA) is a chronic inflammatory rheumatic disease affecting the joints and other organ systems [1]. Accelerated atherosclerosis and increased stroke morbidity and mortality have been associated with RA [2-4], however, no systematic evaluation of intracranial circulation has yet been performed.

Intracranial vessels, primarily the middle cerebral arteries (MCA) are assessed by transcranial doppler (TCD). With this technique, cerebral arteries are studied through relatively thin bone windows [5]. Approximately 8-20\% of healthy individuals have inadequate temporal acoustic windows (TAW) [6-8]. This is also defined as TAW failure (TAWF) [9]. The thickness and texture of temporal bone may also influence TAW detectability [9]. There is an increased prevalence of in TAWF in females and in elderly people [6, 7]. In a stroke study, TCD signals could not be adequately obtained due to TAWF in $34 \%$ of patients. TAWF, bone thickness and inhomogeneous texture correlated with age and with each other [9].

In stroke patients, TAWF did not correlate with vertebral, femoral or total bone mineral density (BMD). However, heterogeneous temporal bone texture correlated with lower BMD [9]. We have not found any reports on possible associations between TAWF, temporal bone texture and thickness and various bone markers.

Here we assessed the availability of TAWs in patients with RA. We used a selected healthy population without any known cardiovascular (CV) risk as control group. We determined TAWF, temporal bone thickness and texture. As RA has been associated with increased bone turnover and generalized bone loss [10,11], which could affect bone deposition around the TAW, we also studied the features of TAW in relation to BMD and bone markers. As the examiner has to examine both left and right cerebral arteries during TCD assessment, it is also important to assess TAW on both sides. Furthermore, there may be anatomical differences between left and right side arteries, therefore, we also wished to compare TAW features on left vs right side. To our knowledge, this is the very first study assessing TAWs, TAWF and characteristics of the temporal bone in RA. 


\section{Patients and methods}

Patients and controls

Sixty-two consecutive RA patients were recruited for the study. The major characteristics of the patients are shown in Table 1. All patients were females, their mean age was 60.7 \pm 9.5 (range: $27-78$ ) years. Mean disease duration was 11.5 \pm 7.6 (range: 1-36) years. Altogether $75 \%$ were IgM rheumatoid factor (RF) positive, and 75\% were anti-CCP positive. Among these patients, 20 were biologic-free. They had been receiving MTX for a mean $6.8 \pm 4.7$

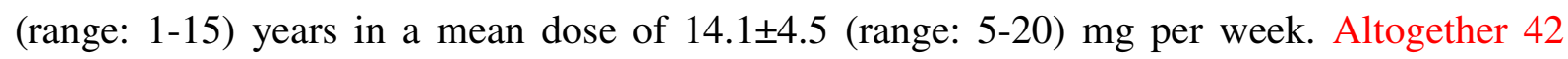
patients had been receiving biologics for a mean duration of $5.2 \pm 1.9$ (range: 1-8) years. In addition, 32 patients were on low-dose corticosteroids. However, they had been receiving it in a stable dose for at least six months.

A cohort of 60 non-RA women were chosen as control group. Their mean age was $56.3 \pm 10.9$ (range: 29-90) years. The existence of TAWF was determined in these subjects. None of these individuals had hypertension, known cardiovascular or cerebrovascular disease. The thickness and bone structure of TAWs were assessed in a subset $(n=35)$ of this control population. In this subset, the mean age was $53.7 \pm 4.0$ (range: $48-60$ ) years.

The Borsod County Teaching Hospital IRB approved this study (No. 13/2016).

Determination of temporal windows

Before the TCD assessment was carried out by ultrasound (not included in this paper), the ultrasonographer (M.S., a single observer) determined whether the right and left TAW were detectable or not. TAWF was determined in 62 RA patients and 60 control subjects. A TAW score was also established. A score of 0,1 or 2 indicated TAWF on both sides, either side or none of the sides, respectively.

Furthermore, the thickness and structure ("condense" cortical bone or "sandwich"-like trilayer) of TAWs were determined by computed tomography (CT) (Somatom Definition AS, 64-slice, Siemens, Germany) in 43 RA patients and 35 healthy controls. The instrument was set 
to bone window and native images of $2 \mathrm{~mm}$ thick slices were evaluated. The thickness of TAW expressed in $\mathrm{mm}$ was measured at a point $3 \mathrm{~cm}$ anterior to the earlobe. With respect to the texture, either a thin temporal bone with a condense, homogenous cortical structure, or a thicker, heterogenous trilayer (cortical-diploe-cortical) pattern could be detected around the TAW [9]. (Bone density was not specifically assessed, however, it is always higher in the compact cortical bone, and lower in the thicker trilayer.)

Bone densitometry and bone markers

BMD and T-scores of the lumbar L1 and L2-4 vertebrae, femoral neck and total hip were assessed by DEXA (Hologic 010-0575, Bedford, MA, USA).

Among bone biomarkers, serum calcium and phosphate were determined by routine laboratory methods. Serum osteocalcin (OC; normal: $<41 \mu \mathrm{g} / 1)$, $\beta$-CrossLaps (CTX; normal: $<0.57 \mu \mathrm{g} / \mathrm{l}$ ), P1NP (normal: <75 $\mu \mathrm{g} / \mathrm{l}$ ), 25-hydroxy-vitamin $\mathrm{D}_{3}(25-\mathrm{OH}$-vitD 3 ; normal: > 75 nmol/l), parathormon (PTH; normal: 1.6-6.9 pmol/1), osteoprotegerin (OPG; median: 2.7 pmol/l, normal: to be determined [TBD] locally in the lab), sclerostin (SOST; median: 24.1 pmol/l, normal: TBD), free soluble RANK ligand (sRANKL; median: $0.37 \mathrm{pmol} / 1$ in females, normal: TBD) were determined by ELISA (Biomedica, Vienna, Austria). OPG/sRANKL ratios were also calculated.

\section{Statistical analysis}

The statistical analysis was processed with IBM SPSS 22 software. The data are expressed as the mean \pm SD and frequencies and percentages. Continuous variables were evaluated by paired two-tailed t-test and Wilcoxon test. Nominal variables were compared between groups using the chi-squared or Fisher's exact test, as appropriate. Simple correlations were determined by Spearman's analysis. Multiple linear regression using the stepwise method was used to determine correlations and independent associations between parameters. TAW parameters were the dependent variables and several other clinical and laboratory parameters were independent variables. The $\mathrm{B}(+95 \% \mathrm{CI})$ regression coefficient indicated independent association between the dependent and independent variable during changes. $\mathrm{P}$ values $<0.05$ were considered significant. The SPSS 22.0 software was used for statistical analysis. 


\section{Results}

Detectability, texture and thickness of TAW in RA versus controls

In RA, 34 out of the 62 patients $(54.8 \%$ ) had TAWF on at least one side. (Neither TAW could be identified in $34 \%$ of RA subjects.) In contrast, only $20.0 \%$ of control subjects had TAWF on either or both sides $(\mathrm{p}<0.001)$ (Table 2).

TAW texture could be assessed in 43 RA patients and 35 controls. In control subjects, only one out of 35 subjects exerted the "sandwich-like" structure on either sides (2.9\%). On the other hand, in RA, $53.0 \%$ of patients exerted this trilayer texture on at least one side $(\mathrm{p}<0.001)$ (Table 2). Thus, while only less than $3 \%$ of healthy subjects have sandwich TAW structure, one-third to more than half of RA patients may exert this feature (Table 2).

Bone thickness around TAW could be assessed also in 43 RA patients and 35 controls. In RA vs controls, the mean temporal bone thickness values of the right TAW were $3.58 \pm 1.43$ $\mathrm{mm}$ vs $2.92 \pm 1.22 \mathrm{~mm}(\mathrm{p}=\mathrm{NS})$, while those of the left TAW were $4.16 \pm 1.56 \mathrm{~mm}$ vs $2.90 \pm 1.16$ $\mathrm{mm}(\mathrm{p}=0.001)$ (Table 2).

Correlations between TAW features and disease markers in RA

First, the various TAW parameters were correlated with each other (Table 3). There have been significant associations between TAWF, thickness and structure. In the RA cohort $(n=62)$, the absence of right TAW was significantly associated with increased thickness $(\mathrm{R}=0.811, \mathrm{p}<0.001)$ and the sandwich-like texture of the right TAW $(\mathrm{R}=0.712, \mathrm{p}<0.001)$. Similar observations were made with respect to the left TAWs (thickness: $R=0.768, \mathrm{p}<0.01$, texture: $\mathrm{R}=0.791, \mathrm{p}<0.01)$. The thickness of right and left TAWs also correlated with the heterogeneous texture on the corresponding sides (right: $\mathrm{R}=0.569, \mathrm{p}<0.01$, left: $\mathrm{R}=0.457$, $\mathrm{p}=0.02)$ (Table 3). 
Binary logistic regression analysis was performed in order to determine correlations between TAW characteristics (dependent variables) and other clinical and laboratory parameters (independent variables) (Tables 4 and 5). In the linear regression analysis, TAWF (lower TAW scores) on both sides $(\mathrm{p}=0.007)$ significantly correlated with age. Interestingly, left TAWF was associated with OPG levels $(\mathrm{p}=0.012)$ (Table 4). In addition, this texture on right $(\mathrm{p}=0.045)$ or left $(\mathrm{p}=0.016)$ side exerted significant correlation with OPG (Table 3). Right $(\mathrm{p}=0.025)$ or left $(\mathrm{p}=0.007)$ TAW bone thickness correlated with age. Finally, TAW thickness on the left side correlated with femoral neck and total hip bone loss (Table 4).

Multiple regression analysis was performed to determine independent prognostic factors for TAWF in RA. Age ( $p=0.026)$ and serum OPG levels $(p=0.012)$ were independent predictors of left TAWF. Age was also a predictor of sandwich-like texture of the left TAW ( $\mathrm{p}=0.007$ ) (Table 5).

\section{Discussion}

TAW accessibility is essential to perform TCD. Unfortunately, in the general population, TAWF is found in about 8-20\% of individuals [6-8]. In stroke patients TAWF may be more common (34\%) [9]. The texture and the thickness of temporal bone may also be of importance. TAWF has been associated with temporal bone inhomogeneity and increased thickness [9]. The characteristics of TAW have not yet been studied in RA patients so we do not have any reported data for comparison.

Here we performed a study carried out on 62 RA patients in comparison to a population of 60 non-RA individuals undergoing routine TCD screening. In RA patients, TAWF was observed on either or both sides in 34-53\% of patients and in 13-20\% of controls. The frequency of TAWF was significantly higher compared to controls and was also higher than what was reported by others in the general population [6].

While only one control subject (3\%) had the heterogeneous temporal bone structure, this phenomenon was observed in 53-60\% of RA patients. Bone inhomogeneity has also been described in stroke patients [9]. 
The temporal bone was also thicker in RA patients (3.6-4.6 $\mathrm{mm}$ ) compared to controls (2.9 mm). Stroke patients may also exert thicker temporal bones [9].

We observed significant paired correlations between TAWF, temporal bone heterogeneous texture and thickness on both sides in RA. Similar observations were reported in patients with acute stroke [9]. By performing linear logistic regression analysis, TAWF, sandwich-like texture and bone thickness all correlated with age. Interestingly, TAWF and the special trilayer texture also correlated with serum OPG levels. OPG was the only bone biomarker that exerted association with TAW characteristics. Finally, left TAW bone thickness inversely correlated with total hip and femoral neck BMD and T-scores indicating, that locally increased TAW thickness may be seen in patients with more pronounced generalized osteoporosis. We also performed multivariate logistic regression analysis. Age was independent prognostic factor for TAWF and sandwich-like texture. Again, serum OPG was an independent predictor of TAWF in RA patients. With age, the temporal bone gets thicker leading to reduced detectability of the foramen. Similar correlations were found in other studies carried out in the general population [6] and in stroke patients [9]. TAW thickness was inversely correlated with hip and femoral neck BMD. RA, especially active disease, has been associated with generalized bone loss [10-13] primarily due to inflammatory bone resorption [14, 15]. In stroke patients, there was no association between TAWF and BMD, however temporal bone heterogeneity, similarly to our results, was inversely associated with BMD [9]. OPG is a decoy receptor and inhibitor of RANK-RANKL-mediated inflammatory bone loss [16]. In RA, OPG production may be a counter-regulator of bone resorption $[16,17]$ and both anti-TNF [18] and anti-IL-6 biologics [19] increase circulating OPG levels. In our study, TAWF and heterogeneous texture were correlated with higher OPG levels.

In conclusion, TAWF is more prevalent in RA patients than in the general population. We can hypothesize that as TAWF and TAW thickness have been associated with increased production of OPG and lower hip BMD and T-score, respectively, it is likely, that secondary generalized osteopenia/osteoporosis observed in a great proportion of RA patients, especially in those with more severe disease $[10,11]$ may induce the counter-regulatory release of OPG. OPG, in turn, may also stimulate bone apposition in the temporal bone surrounding the TAW leading to TAWF, increased thickness and, due to more rapid burn turnover, heterogeneous bone texture $[18,19]$. Clinically, the detection of TAW is not only important to understand temporal bone homeostasis that may also reflect systemic bone metabolism, but also to select patients suitable for TCD assessments. More studies to be carried out in large number of patients 
are necessary to further characterize TAWF, features of the temporal bone and their relation to bone biology.

\section{Acknowledgements}

The work was supported by the European Union and the State of Hungary co-financed by the European Social Fund in the framework of TAMOP-4.2.4.A/2-11/1-2012-0001 'National Excellence Program'(Z.S.), by research grant TÁMOP-4.2.2.A-11/1/KONV-2012-0031 financed by the European Union (Z.S.); an OTKA K10073 grant (H.P.B and Z.S.) donated by the Hungarian Scientific Research Fund; and also by the European Union grants GINOP-2.3.215-2016-00015 and GINOP-2.3.2-15-2016-00050 (Z.S.).

\section{Ethics, consent and permissions, consent to publish}

Ethical approval see above (Patients and methods).

All patients gave informed consent to the study and all of them gave consent to publish. 


\section{References}

1. Symmons DP: Epidemiology of rheumatoid arthritis: determinants of onset, persistence and outcome. Best Pract Res Clin Rheumatol 2002, 16(5):707-722.

2. Shoenfeld Y, Gerli R, Doria A, Matsuura E, Cerinic MM, Ronda N, Jara LJ, Abu-Shakra M, Meroni PL, Sherer Y: Accelerated atherosclerosis in autoimmune rheumatic diseases. Circulation 2005, 112(21):3337-3347.

3. Szekanecz Z, Kerekes G, Vegh E, Kardos Z, Barath Z, Tamasi L, Shoenfeld Y: Autoimmune atherosclerosis in 3D: How it develops, how to diagnose and what to do. Autoimmun Rev 2016, Epub 2016 Mar 12.

4. Kerekes G, Szekanecz Z, Der H, Sandor Z, Lakos G, Muszbek L, Csipo I, Sipka S, Seres I, Paragh $G$ et al: Endothelial dysfunction and atherosclerosis in rheumatoid arthritis: a multiparametric analysis using imaging techniques and laboratory markers of inflammation and autoimmunity. J Rheumatol 2008, 35(3):398-406.

5. Guan J, Zhang S, Zhou Q, Li C, Lu Z: Usefulness of transcranial Doppler ultrasound in evaluating cervical-cranial collateral circulations. Interventional neurology 2013, 2(1):8-18.

6. Marinoni M, Ginanneschi A, Forleo P, Amaducci L: Technical limits in transcranial Doppler recording: inadequate acoustic windows. Ultrasound Med Biol 1997, 23(8):1275-1277.

7. Bazan R, Braga GP, Luvizutto GJ, Hueb JC, Hokama NK, Zanati Bazan SG, de Carvalho Nunes HR, Leite JP, Pontes-Neto OM: Evaluation of the Temporal Acoustic Window for Transcranial Doppler in a Multi-Ethnic Population in Brazil. Ultrasound Med Biol 2015, 41(8):2131-2134.

8. Postert $\mathrm{T}$, Federlein $\mathrm{J}$, Przuntek $\mathrm{H}$, Buttner $\mathrm{T}$ : Insufficient and absent acoustic temporal bone window: potential and limitations of transcranial contrastenhanced color-coded sonography and contrast-enhanced power-based sonography. Ultrasound Med Biol 1997, 23(6):857-862.

9. Kwon JH, Kim JS, Kang DW, Bae KS, Kwon SU: The thickness and texture of temporal bone in brain $\mathrm{CT}$ predict acoustic window failure of transcranial Doppler. J Neuroimaging 2006, 16(4):347-352.

10. Deal C: Bone loss in rheumatoid arthritis: systemic, periarticular, and focal. Curr Rheumatol Rep 2012, 14(3):231-237.

11. Schett G, Saag KG, Bijlsma JW: From bone biology to clinical outcome: state of the art and future perspectives. Ann Rheum Dis 2010, 69(8):1415-1419.

12. Suzuki Y, Mizushima Y: Osteoporosis in rheumatoid arthritis. Osteoporos Int 1997, 7 Suppl 3:S217-222.

13. Rass P, Pakozdi A, Lakatos P, Zilahi E, Sipka S, Szegedi G, Szekanecz Z: Vitamin D receptor gene polymorphism in rheumatoid arthritis and associated osteoporosis. Rheumatol Int 2006, 26(11):964-971.

14. Schett G: Osteoimmunology in rheumatic diseases. Arthritis Res Ther 2009, 11(1):210.

15. Schett G, Gravallese E: Bone erosion in rheumatoid arthritis: mechanisms, diagnosis and treatment. Nat Rev Rheumatol 2012, 8(11):656-664.

16. Schett G, Redlich K, Hayer S, Zwerina J, Bolon B, Dunstan C, Gortz B, Schulz A, Bergmeister $\mathrm{H}$, Kollias $\mathrm{G}$ et al: Osteoprotegerin protects against generalized bone loss in tumor necrosis factor-transgenic mice. Arthritis Rheum 2003, 48(7):20422051.

17. Zwerina J, Hayer S, Tohidast-Akrad M, Bergmeister H, Redlich K, Feige U, Dunstan C, Kollias G, Steiner G, Smolen J et al: Single and combined inhibition of tumor 
necrosis factor, interleukin-1, and RANKL pathways in tumor necrosis factorinduced arthritis: effects on synovial inflammation, bone erosion, and cartilage destruction. Arthritis Rheum 2004, 50(1):277-290.

18. Wang SY, Liu YY, Ye H, Guo JP, Li R, Liu X, Li ZG: Circulating Dickkopf-1 is correlated with bone erosion and inflammation in rheumatoid arthritis. $J$ Rheumatol 2011, 38(5):821-827.

19. Terpos E, Fragiadaki K, Konsta M, Bratengeier C, Papatheodorou A, Sfikakis PP: Early effects of IL-6 receptor inhibition on bone homeostasis: a pilot study in women with rheumatoid arthritis. Clin Exp Rheumatol 2011, 29(6):921-925. 
Table 1. General characteristics and laboratory markers of RA patients

\begin{tabular}{|c|c|}
\hline & RA patients \\
\hline $\mathrm{n}$ & 62 \\
\hline age (years) & $60.7 \pm 9.5$ \\
\hline disease duration (years) & $11.5 \pm 7.6$ \\
\hline RF positivity (\%) & $45(74 \%)$ \\
\hline anti-CCP positivity (\%) & $30(75 \%)$ \\
\hline MTX duration (years) & $6.5 \pm 3.9$ \\
\hline MTX dose (mg/week) & $14.9 \pm 5.5$ \\
\hline Low-dose corticosteroid (n) & 32 \\
\hline Biologic-treated (n) & 42 \\
\hline MTX-treated (n) & 20 \\
\hline biologic duration (years) & $5.2 \pm 1.9$ \\
\hline DAS28 & $2.46 \pm 0.82$ \\
\hline ESR $(\mathbf{m m} / \mathbf{h})$ & $17.42 \pm 14.50$ \\
\hline hsCRP (mg/l) & $5.35 \pm 7.83$ \\
\hline L1 BMD $\left(\mathrm{g} / \mathrm{cm}^{2}\right)$ & $0.872 \pm 0.131$ \\
\hline L1 T-score & $-1.25 \pm 1.18$ \\
\hline L2-4 BMD $\left(\mathrm{g} / \mathrm{cm}^{2}\right)$ & $0.922 \pm 0.132$ \\
\hline L2-4 T-score & $-1.13 \pm 1.24$ \\
\hline Femoral neck BMD $\left(\mathrm{g} / \mathrm{cm}^{2}\right)$ & $0.698 \pm 0.118$ \\
\hline Femoral neck T-score & $-1.41 \pm 0.94$ \\
\hline Total hip BMD $\left(\mathrm{g} / \mathrm{cm}^{2}\right)$ & $0.825 \pm 0.140$ \\
\hline Total hip T-score & $-0.92 \pm 1.22$ \\
\hline osteocalcin $(\mathrm{OC}, \mu \mathrm{g} / \mathrm{l})$ & $18.53 \pm 6.57$ \\
\hline$\beta-\mathrm{CTX}(\mu \mathrm{g} / \mathrm{l})$ & $0.302 \pm 0.140$ \\
\hline P1NP $(\mu \mathrm{g} / \mathrm{I})$ & $50.74 \pm 18.70$ \\
\hline 25-OH-vitD3 $(\mathrm{nmol} / \mathrm{l})$ & $58.20 \pm 28.16$ \\
\hline PTH-I (pmol/l) & $4.14 \pm 1.58$ \\
\hline sclerostin (SOST, pmol/l) & $31.99 \pm 10.52$ \\
\hline osteoprotegerin (OPG, pmol/l) & $7.49 \pm 3.35$ \\
\hline SRANKL (pmol/l) & $0.127 \pm 0.102$ \\
\hline OPG/RANKL ratio & $214.16 \pm 620.80$ \\
\hline
\end{tabular}


Table 2. TAW features in RA patients and controls

\begin{tabular}{|c|c|c|c|}
\hline & RA patients & Controls & $\mathbf{p}$ \\
\hline TAW failure on either side $(\%)$ & 54.8 & 22.0 & $<0.001$ \\
\hline „Trilayer" (sandwich) texture & 53.0 & 2.9 & $<0.001$ \\
\hline TAW thickness - right (mm) & $3.58 \pm 1.43$ & $2.92 \pm 1.22$ & NS \\
$-\quad$ left $(\mathrm{mm})$ & $4.16 \pm 1.56$ & $2.90 \pm 1.16$ & 0.001 \\
\hline
\end{tabular}


Table 3. Correlations between various TAW features in RA patients $(n=62)$

\begin{tabular}{|c|c|c|c|}
\hline Parameter 1 & Parameter 2 & $\mathbf{R}$ value & p value \\
\hline \multicolumn{4}{|c|}{ Right TAW } \\
\hline TAWF & thickness & 0.811 & $<0.001$ \\
\hline TAWF & heterogeneous texture & 0.712 & $<0.001$ \\
\hline thickness & heterogeneous texture & 0.569 & $<0.001$ \\
\hline \multicolumn{4}{|c|}{ Left TAW } \\
\hline TAWF & thickness & 0.768 & $<0.001$ \\
\hline TAWF & heterogeneous texture & 0.791 & $<0.001$ \\
\hline thickness & heterogeneous texture & 0.457 & 0.002 \\
\hline \multicolumn{4}{|c|}{ Right vs Left TAW } \\
\hline TAWF (R) & TAWF (L) & 0.634 & $<0.001$ \\
\hline thickness (R) & thickness (L) & 0.754 & $<0.001$ \\
\hline texture $(\mathrm{R})$ & texture $(\mathrm{L})$ & 0.721 & $<0.001$ \\
\hline
\end{tabular}


Table 4. Correlations between TAW features and other characteristics in RA patients $(\mathrm{n}=62)^{*}$

\begin{tabular}{|c|c|c|c|}
\hline & \multicolumn{3}{|c|}{ Odds $[\operatorname{Exp}(B)](C I) 95 \%)$ p value } \\
\hline side & right & left & both \\
\hline \multicolumn{4}{|c|}{ TAW failure/score } \\
\hline age (years) & $1.1(1.025-1.181), \mathrm{p}=0.008$ & $1.190(1.079-1.311), \mathrm{p}<0.001$ & $1.106(1.028-1.189) \mathrm{p}=0.007$ \\
\hline osteoprotegerin (OPG, pM) & & $1.690(1.123-2.542), \mathrm{p}=0.012$ & \\
\hline \multicolumn{4}{|c|}{ TAW texture (sandwich-like) } \\
\hline age & & $0.081(0.023-0.140), p=0.007$ & - \\
\hline osteoprotegerin (OPG, pM) & $0.807(0.647-1.005), p=0.045$ & $0.653(0.462-0.922), p=0.016$ & - \\
\hline \multicolumn{4}{|c|}{ TAW thickness } \\
\hline age & $0.063(0.008-0.118), p=0.025$ & $0.081(0.023-0.140), p=0.007$ & - \\
\hline femoral neck BMD $\left(\mathrm{g} / \mathrm{cm}^{2}\right)$ & & $-5.708(-9.995--1.420), p=0.010$ & - \\
\hline femoral neck T-score & & $-0.633(-1.109--0.157), p=0.010$ & - \\
\hline total hip BMD $\left(\mathrm{g} / \mathrm{cm}^{2}\right)$ & & $-4.037(-7.110--0.963), p=0.011$ & - \\
\hline total hip T-score & & $-0.493(-0.871--0.116), p=0.012$ & - \\
\hline
\end{tabular}

${ }^{*}$ Linear binary logistic regression. Table only shows statistically significant correlations. See text for abbreviations. 
Table 5. Independent prognostic factors for TAW failure in RA $(n=62)^{*}$

\begin{tabular}{|c|c|c|c|}
\hline & \multicolumn{3}{|c|}{ Odds $[\operatorname{Exp}(B)](C I 95 \%), p$ value } \\
\hline side & right & left & both \\
\hline \multicolumn{4}{|c|}{ TAW failure/score } \\
\hline age (years) & & $1.531(1.051-2.231), \mathrm{p}=0.026$ & \\
\hline osteoprotegerin (OPG, pM) & & $1.154(1.005-1.325), \mathrm{p}=0.012$ & \\
\hline \multicolumn{4}{|c|}{ TAW texture (sandwich-like) } \\
\hline age & & $0.081(0.023-0.140), p=0.007$ & - \\
\hline
\end{tabular}

${ }^{*}$ Multivariate binary logistic regression. Table only shows statistically significant correlations. See text for abbreviations. 